\title{
La soudure électrique et les conduites forcées
}

\section{Electric welding and penstocks}

\author{
P'AR (i. FERRANI)

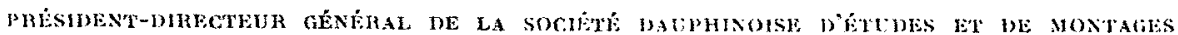

\begin{abstract}
l'application industrielle du procédé de soudur" à l'arc electrique a profondément modifié et perfectionné la technique de la chaudronnerie en géneral et celle des conduites forcies en particulier.

Elle a paisiamment contribue à lamélioration ale la qualifi, du fini et de la présentation des appareils.

La soudure électrique a permis la mise en arubre des aciers à hanle limite elas/ique que les procides anlirieurs ne permeltaient pas de sonder. Résume's d'essitis sur ariers a molpenne leneul en arbone COF: (on CONFOR E). Vérificalion de tenr sondabilité et de leur sécurile diutilisation.
\end{abstract}

Les possibilités que la soudure électrique a permises dans la construction des conduites forcées de hautes chutes à grande puissance des usines hydro-cleetriques modernes sont considérables.

Lat soudure álectrique a en effet remplacé en matiere de construction de conduites forcées la soudure au gaz à l'eau utilisée à la fabrication de luyaux de grands diametres qui n'avait son emploi que sur des tóles en acier extra-doux à 4) $\mathrm{kg} / \mathrm{mm}^{2}$ de résistance maximum.

Avec la soudure électrique on soude maintenant sans difficulté en toute sécurité, avec une sarantie de $100 \%$ de la résistance de la soudure par rapport à celle du métal de base, des aciers demi-dur's dont l'emploi a permis d'importants progris dans la fabrication des tuyaux auto-fretlés et de réaliser les luyaux surpressés.

Les tuyaux « auto-frettés » ainsi que les Luyaux «surpressés» sont de plus en plus utilisós sur les conduites forcées alimentant les usines hydro-clectriques modernes de grande puissance lonctionnant sous de hautes chutes, et lorsqu'il

\begin{abstract}
Industrial use of the chectris-are welding process has greatly modified and improned the techniques of metal working in general and of penstoclis in particular. It has contributed greally to improvements in the quality, finish and presentation of the apparatas. Electric anelding has allowed the use of stepls habing high elastie limils which could not be welded by the melhods ased prenionsly. sumumary of tests on steots hubing an aberalge rorbon conlent COF :3 (or CONFOR E). Che king their adoplability to medtin! and the reliabilit! in 1 sse.
\end{abstract}

s'agit de tris hautes chutes, on utilise leurs dirivés: les "tuyaux surpressés aulo-frettés of les « tuyaux auto-freltés surpressés ».

La construction des \& hyaux surpressés \& est basée sur le principe bien connu du relevement de la limile élastique d'un acier par cerouissasge sous fraction à froid.

D'une maniere generale, l'cerouissage se traduil par une augmentation de la charge de ruplure et par une élévation plus grande encore do la limite élastique. Cette operation est particulicrement avantageuse, puisqu'elle permet d'élrever la charge utile d'une pieco sans rendre pour cela son emploi dangerenx; un sait en efret qu'un acier écroui par traction ne devient ni cassant, ni fragile, ni hétérogene. La longue expópience que l'on a maintenant de l'utilisation des aciers soudables etirés à froid suffit a démontrer que re procede améliore la qualiti ef la sécurité des ourages sur lesquels il est appliqué. Si l'on compare deux aciers du même type (au carbone par exemple) de mème limite élastique, l'un normalisé de nuance plus dure que l'autre dont la li- 
mile elostique a ble relever par icrouissisge, on constate pour celui-ci une resilience et un allongement supérieurs à ceux du premier.

Loin de redouter l'écrouissage, on l'applique. systématiquement, pour amćliorer les proprićtés mécaniques des metaux, aux ronds pour beton armé par exemple, que lon tractionne bien audessus de leur limite alastique ef parfois mène jusquau moment oi samorce la striction.

C'est lutilisation de cette propricite ef son application a la construction de tuyaux destincs a supporter des pressions intérieures élevées qui a donné naissance aux «tuyaux surpressés».

Pour définir les tuyaux "surpressés aulolrellés ot les "tuyaux auto-frettés surpressés», il suffil de se rappeler ce que sont les « luyaux aulo-frellés 》 qui, depuis 1925, équipent toutes les haules chutes frimçaises à grande puissance.

Les premiers ne sont autres que des tuyaux auto-frettes dont la paroi est préalablenent surpressee, les seconds sont des tuyaux auto-frettés dont la paroi et les frettes sont simultaniment soumises à l'opération de surpressage.

La nouveaulé des tuyaux * auto-frettés surpressés $\gg$ réside dans le fait que jusqualors on arait systématiquement évité d'atteindre la limile elastique des frettes au cours des opérations d'auto-fretlage, alors que maintenant on procede systématiquement à l'écrouissage des frelles par lraction a froid.

Nous ne reviendrons pas sur te mode de construction ni sur l'interêt pratique el économique des tuyaux surpressés, en priant nos lecteurs de se reporter at la communication que nous avons faite it ce sujet au 4" Congres International des Fabrications Mécaniques à Stockholm en 1952; mais nous attirons leur attention sur la partie de cette communication ou nous faisons ressorlir un avantage très important du surpressage sous l'angle de la relaxation des tensions internes conséculives à l'exécution des soudures (*).

Il est bon de rappeler en passant que les luyaux surpressés sont exempts de fragilité à toules tempéralures aussi bien pour la soudure ellemème que pour le métal de base, et que les marges dallongement apres écrouissage et vieillissement artificiel sont importantes.

Les eritiques de gens mal informes ou de prom lames en la matière n'ont pas dé ménagées à la lechnique du surpressage qui ne s'applique, 't nous insistons sur ce point, qu'à des conduiles forcées fonctionnant sous températures humaines, of non a des apparcils thermigues fonclionnant sous températures élevées.

D'ailleurs, les aciers utilisés à la fabrication

\footnotetext{
(*) Voir la reproduction integrale qui a eté faite de cotte communication dans la revue la Mnalle Manche. 112, miा $19 \% 3$
}

des luyalux surpresses sont non setulement roisilients, mais ils sont aussi non vicillisants.

Pour illustrer ces affirmations, nous pourrions présenter à nos lecteurs les résultats d'innomhrahles essais systématiques faits par de grandes aciéries francaises telles que «Usinor » $\mathrm{el}$ * Longwy , qui sonl les principaux fournisseurs de l'acier "Confor $\mathbf{E}$ » (que nous utilisons pour fabriquer nos conduites forces, mais nous arons sous les yeux une communication presenté par M. l'Ingénieur Curio Fonsaci, Chef mitallurgiste aux Aciéries de la Société Terni à Terni (Italie), au $14^{\circ}$ Congrès International des Centres d'Information de l'Acier. Celle-ci sc rapporte à des aciers dínommés COF. 3 en Ilalie, identiques aux aciers dénommés Confor $E$ ch France. Les essais faits en Italie sur te COF.: sont en tous points eomparables a ceux faits on Frince sur le «Confor $E$ ».

Cette nuanes d'acier est principalement ulilise en ltalie a la fabrication de libles destinces a la construction de tuyaux soudes, auto-fretles ef surpressés pour conduites forées.

Avec lautorisation de M. Fomxica, nous donnons ei-dessous un résumé des essais faits sur COF. 3 pour verifier la sondabilite de le eomportement, à des temperatures normales el all-dessous de xúro, de l'acier préalablement soumis à des opérations d'étirage à lroid, pour montrer la parfaite sécurite dans son utilisation ef laulaptabilite aux diberents besoins de cel areier a moyenne teneur en carbone.

Les resultats montrent a quel point il est possible de eompter, pour les constructions soudies, sur une telle nuance d'acier, alors que quinze amnées se sont écoulies depuis qu'il ful atilisti pour la premiere fois dans une installation importante d'aménagement de conduites forcées.

Pour les usages spéciaux auxquals ils sont deslinés, ces aciers doivent répondre à la nécessité datteindre des ahjectifs techniques ef deonomiques que l'on ne peul atleindre avee des aciers ordinaires à haute teneur en carbone. La construction des conduiles forcies aver ees lypes d'acier entraine le probleme de sécurilé duranl loute leur existence, malgré l'écronissage systématicue a froid du metal de base el des joints soudes. Ces exigences ont conduit is ambiorer nécessairement la qualite, de maniere lolle que l'utilisation de ces aciers ne cossera de s'étendre.

Les résultats des essais dfertués ont toujours blé supérieurs aux minima des spécifications.

Pour domer une ide du slandard moven de quatite de lacier COF. 3, on a lait des diagrammes dans lesquels sont illustrés la répartition en poureentage, la courbe en cloche des valeurs de E (limite clastique) et de R (limite de ruplure) et les courbes de fróquence des propriétés mearnirques de lobles de o à 38 mm d'épaisseur apparlentull at 118 coulees. Lal régularite de ces comb- 


\section{Caractéristiques mécaniques (sens transversal) des tôles COF 3 normalisées}

Epaisseurs 6 a $38 \mathrm{~mm}$ (78. libles de 118 eoulesi

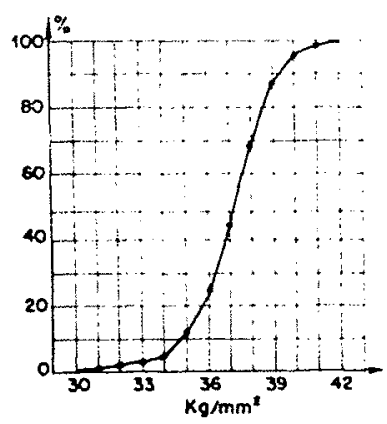

Courbe de distribution en ? des valeurs de t:

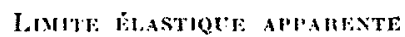

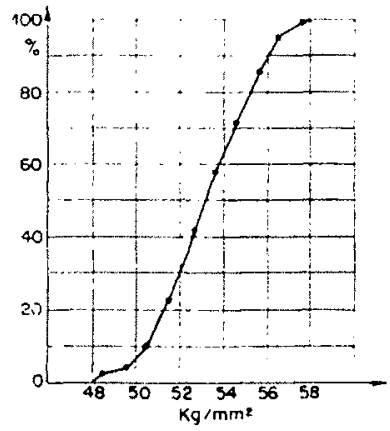

Contribe de distribution

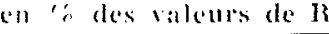

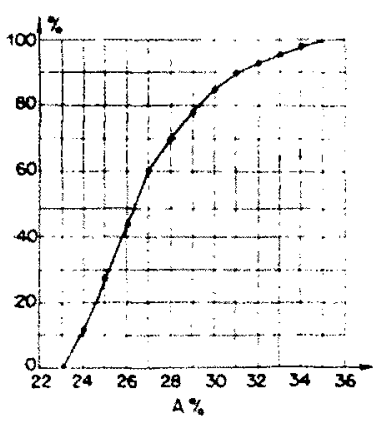

Coube de distribution en ri des valkurs de Ap 7.5

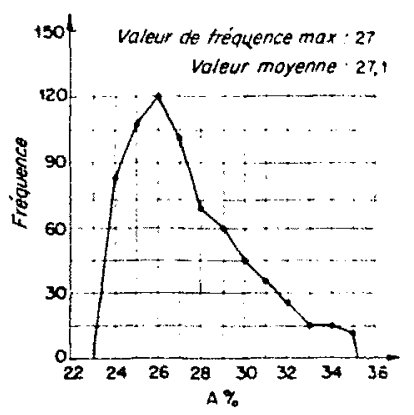

Courbe de tricupenet des valeurs de $A p \pi, \bar{z}$

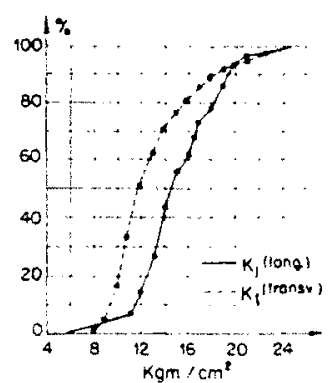

Courbe de distribution ('II ' der valeurs de k

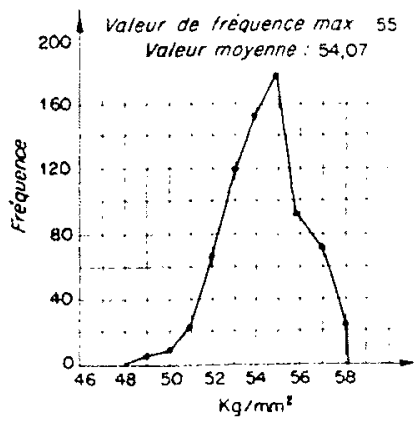

Couble de Trequence des valeurs de R 
Caractéristiques mécaniques des soudures.

Eprouvettes pour l'exécution de joints soudés de tuyaux de conduites forcées.

216 éprouvettes essayées. Sur chatge éprouvette : 2 essais de pliage; 1 essai de traction:

1 essai de reisilience en p!cine soudure;

1 essai de resilience en zone de transition.

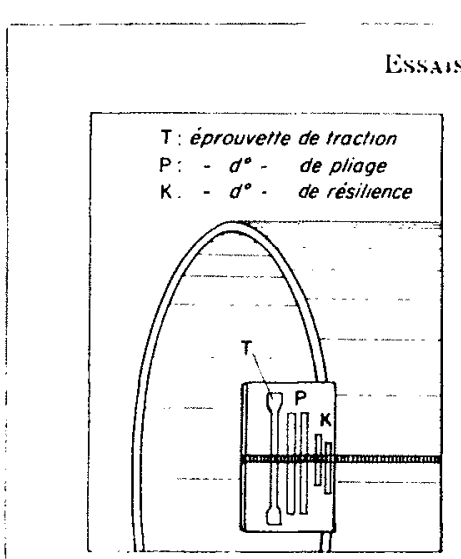

Schéma

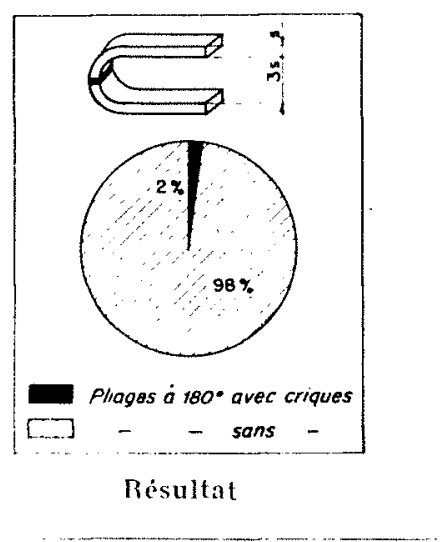

sol Valeur de fréquence max $7 \mathrm{kgm} / \mathrm{cm}^{\prime \prime}$ valeur mayeme $13,2 \mathrm{kom} / \mathrm{cm}^{2}$

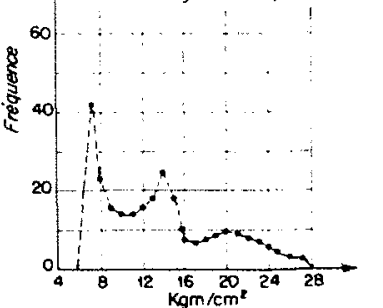

Courbe de fréquence des valeurs de li

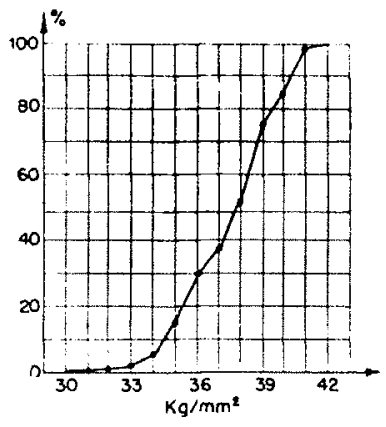

Courbe de distribution en \% des valeurs de
75 voleur dieguence max $: 38,5$ velour moyanno 36,5

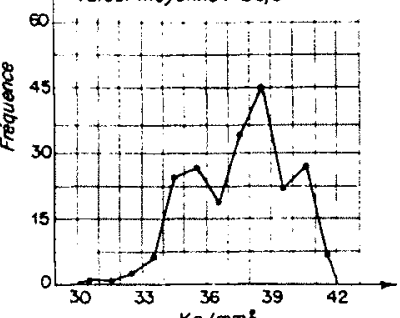

Courbe de réquence des valeurs de 1

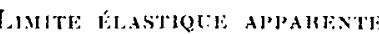

Courbe de $\mathrm{Kam} / \mathrm{cm}^{2}$ istribution en $\%$ des valeurs de $K$

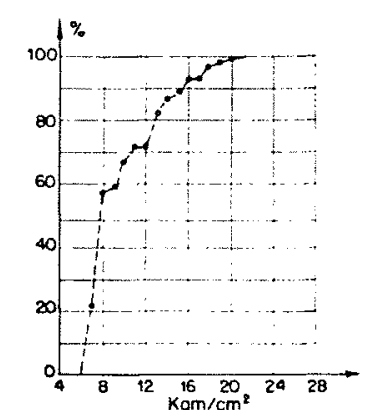

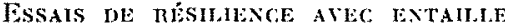
EN PLEINE SOT'NUTE

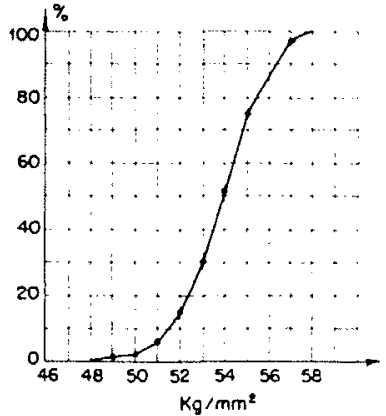

Courbe de distribution en "f des valeurs de 1 REsistavee a La merture

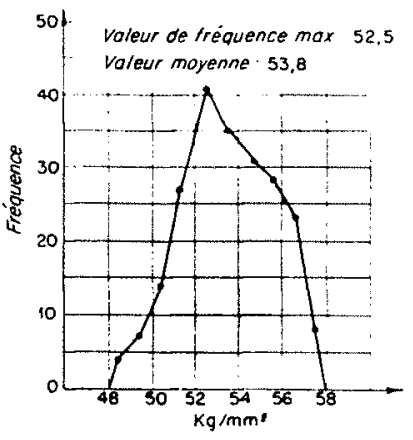

Courbe de rieguence des valeurs de $R$

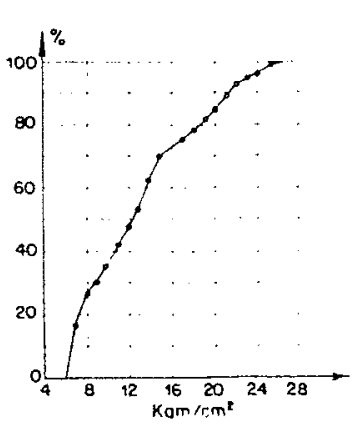

Courbe de distribution en co des valeurs de $k$

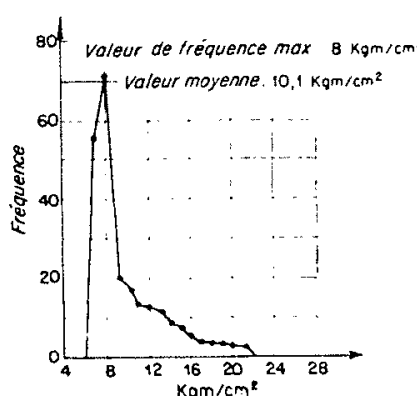

Courbe de fréquence des valcurs de $l i$ 
bes est notable, en comsiderant to fait que quelyues données furent recuedilies an debul de lélahoration de celle nuance d'areier.

les lobes examineses furent presque onlierement utilisés a la construclion de luyaus do conduiles foreces. Apres lepreuve babiluelle en laboraloires des propricles mecaniques, composition el soudabilili, elles furenl a mouveau conIrolies au hasard pour sassurer de la perfection des joints soudes. Ios cepromeltes dessai daient joinles aux tuyaux terminés. I) ecte facon, il i éte possible de recueillir en un lomps remarquablement court un nombre suffisant de donnees pour un examen statistigue, font l'interel réside dans le fait que les domnés relatés sonl plus representalives fue celles oblenues aver des procedés de laboratoỉres. Ie's résullats oblenus sur des cprouveltes dalelier représentent ren fail le standard recel de qualite de la production industrielfe où souvent le travail a lien en lrois equipes, el où l'operalion de somblure esl alecomblic, selon les cireonstances, a la main ou a la somdure antomatique.

Les diagrammes representalifs des rosultals des essais de pliage dans la soudue ont permis de noter quils sont pleinement satisfaisants.

Les diagrammes relatifs à la réporilion pour cont el la frépuence de $F$ of $R$ sur ciprourelles soudés, bien que contenant plus d'irregubariles du fait de leur petit nombre, présentent uno grande ressemblance arec les diagrammes relatifs at motal de hase. Il est interessant de noter yu'ils onl des destes similaires de dispersion. Cela prouve me l'iprouvetle cassait toujours en dehors de la soudure ot ecei est indubitablement un point en faveur de l'uniformite des propriclis mécanicfues el de la lechnicue de sondure.

In diagramme represente le poureentage et la friquener des résullats des essais de resilience; deux autres se rapporlent a des éprouvelles de soudure. Tes essais correspondants furent fails en vue de donner des renseignements sur le stanfard de perfection du melal de soudure ef de lexceution de la soudure.

Deux autres diagrammes eneore se rapportent a des eprouvettes prises dans la zone de transilion de la soudure, domnant ainsi une idée de laptitude de l'acier COF. 3 a supporter les efforts thermiques et mécaniques produits en cours de soudure. Ia diagramme de fréquence de ces iprouveltes a un aspect semblable à celui qui serail oblenu en éprouvant au choc un mćtal de soudure. La similitude est remarquable, specialement par rapport aux limites de dispersion inférieures. On peut noter enfin que les valeurs dans la zone de transition sont, en moyenne, tres proches des valcurs trouvées sur la lible ellemême.

On cxamina igalement le comportement de lacier cor. 3 dans la proxinile immodiale des soudures. Plusieurs soudures sur des toles de dilferentes épaisseurs faites avec le procede dare submerge, ont été soumises à des examens micro el macrographiques. Aucun de ces examens n'a revile de fissures, fólutes ou ha presenee de eomsliluants fragiles. Des essats de fibure furent aussi fails dans le but dexaminer les elfols de la soudure a la main; ils onl permis de constatfer une execllente uniformile de la durele.

Jes aciers COF. 3 sont elabores, ainsi que nous l'avons dejà dil, pour la mise en oeuve de luyaux destines a des conduites forces qui ne subissent bas d'elrorls allernés en servec normal, mais seulement des efrorls ondules de lris faible ammplitude. Elles ne travallent done pas à la faligue ou i l'endurance au sens oì on le comprend senciralloment.

Malgre cola, des essais a la laligue on a lenfurance en nombre limite ont ble effecturs. Ites valeurs des limiles de falligue se sonl toules revelees nellemenl supericures aux valeurs des far ligues maxima envisaseres comme pouvaml alecirembellement se produire an eours d'exploilation.

En service normal, la temprealure minimum d'une conduite forece ne peul descendre en dessous de 0" C, ni dépasser la lempérature maximal de leau vóhiculée. Malgre cola, les proprielis des aciers on guestion ont ale verifiess a hasses et haules temperatures. I.es risultats oblenus sont loul a fail remarefuables.

C'est ansi que l'on a conlrole soignonsomenl

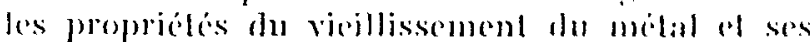
chels a lemperature normale a antedessous de zéro. On a ainsi eomitate que le standarel moven de qualife est remarguable. Cosest bien re jui ressorl de lexamen de diagrammes a leois dimensions illustrant les resullats de piusieme milliers d'essais.

Certains diagrammes interprolent les popriciles do choce el do fartion hansversale des loles

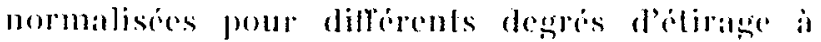
frobl faver on sans vieillissement allificiell solls lemperalures dessai. Lólimese a froid ful oblenu par allongements jusqu'a $10 \%$. Les essais furent faits sous lompópalures comprises entre $-20^{\circ}$ al $240^{\circ} \mathrm{C}$ :

Lobservation de ces diagrammes monlre que la valeur do $\mathrm{E}$ angmente en meme lemper que

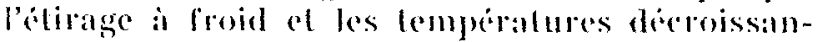
les. Linfluence du rieillissement devient prespue ronstante pour une deformation de $2 \%$ atvere une diminution legere lorsque la temperature s'abaisse.

Les diagrammes d'allongement ont ividemment une direction opposic a coux de traction. On peut noter cependant que même dans los conditions les plus defavomaloles d'erouissage ef de lemperature, les valeurs d'allonsement reslent 
Tôle d'acier COF.3 normalisée de $30 \mathrm{~mm}$ d'épaisseur soudée par le procédé de l'arc submergé.

Chanferin it 40 ;

Soudure secondate à la main;

Soudure principale à la machine automatique \& Vnionmell p:

pas de tratement thermique apres soudure.

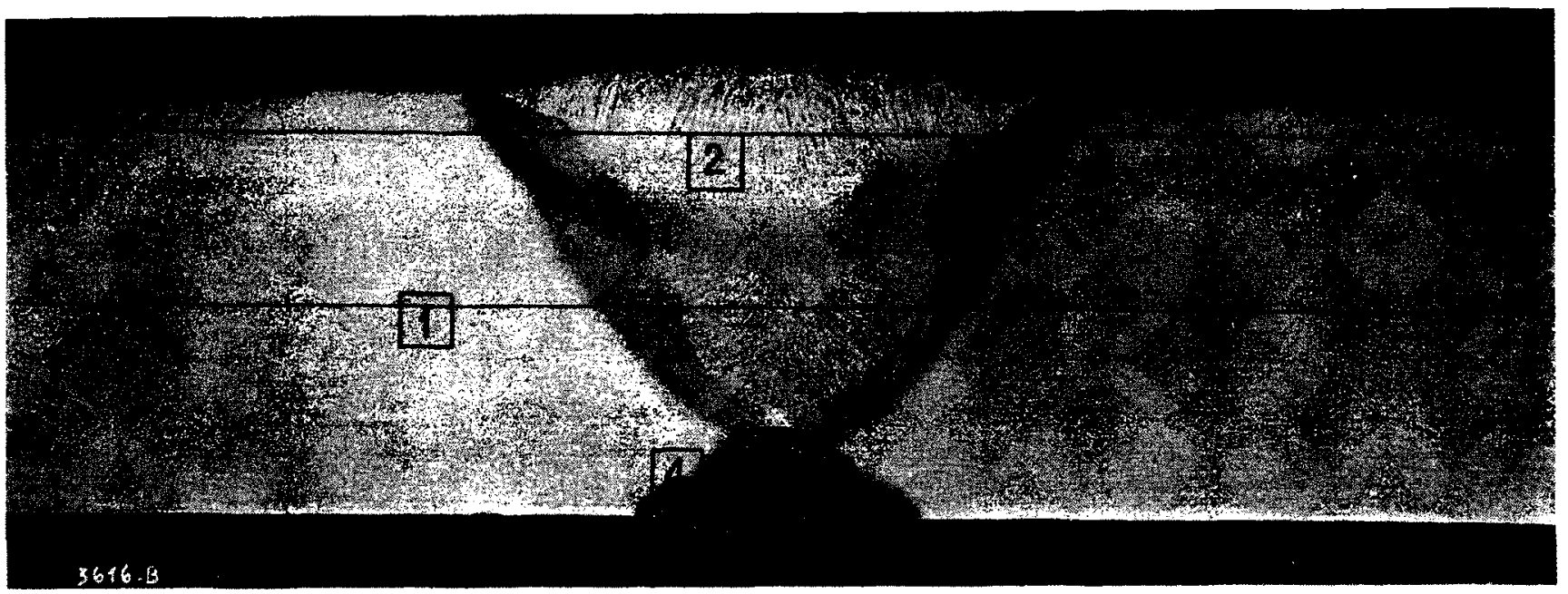

EXAMEN MacrographIOUE
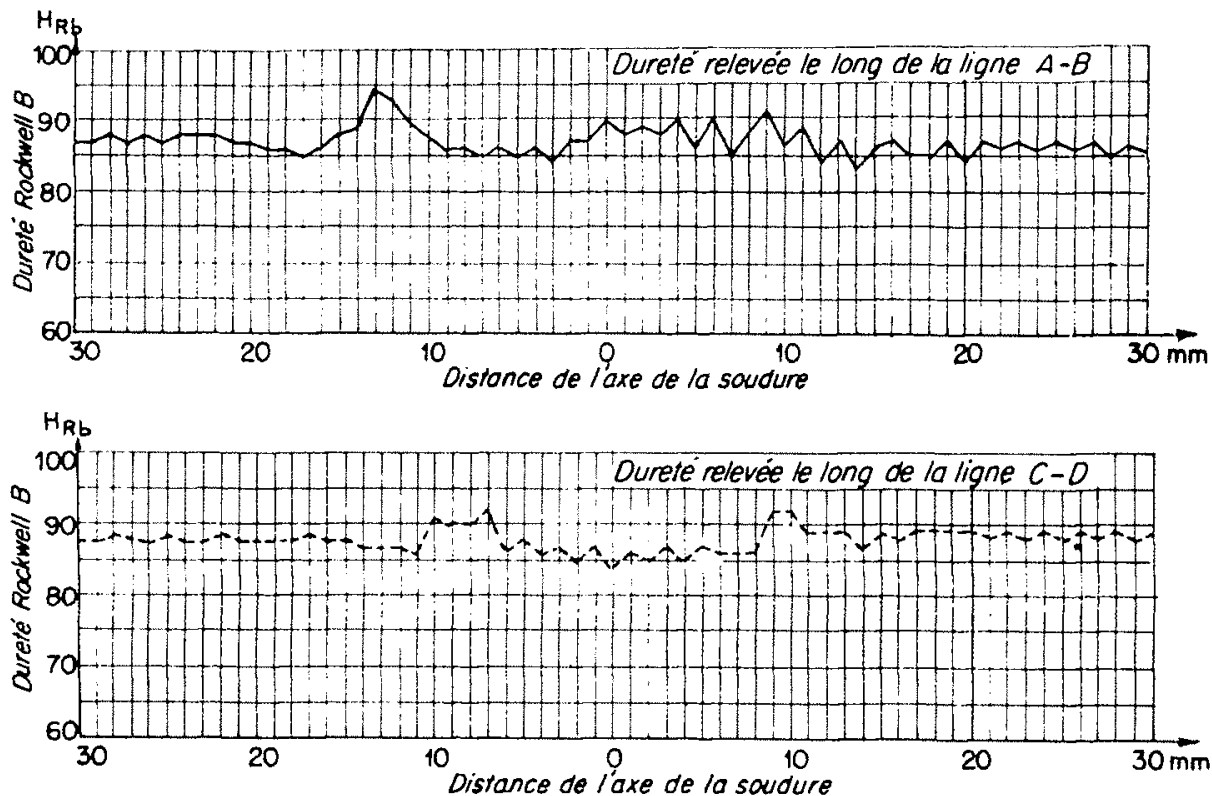

Essais DE Duntit: 
Tóle d'acier COF.3 normalisée de $30 \mathrm{~mm}$ d'épaisseur soudée par le procédé de l'arc submergé.

(C). Tableall 111 ,

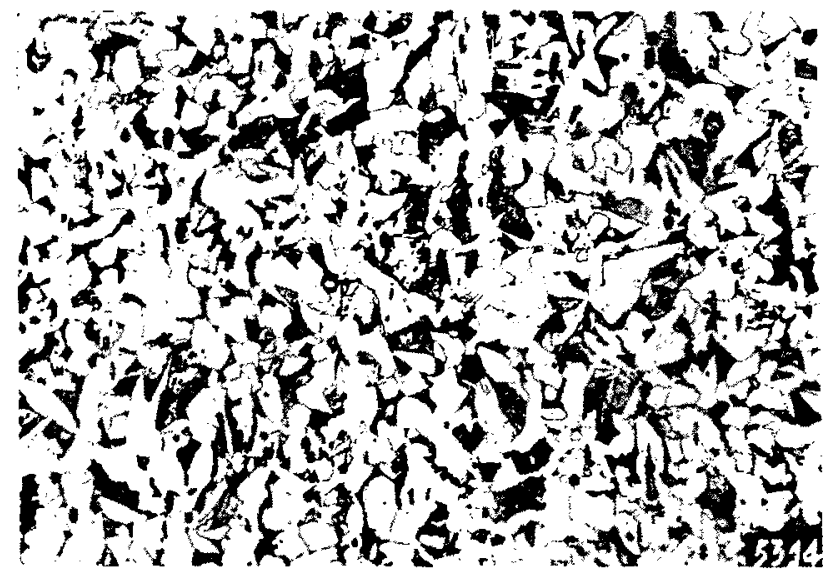

META BH: Bas:

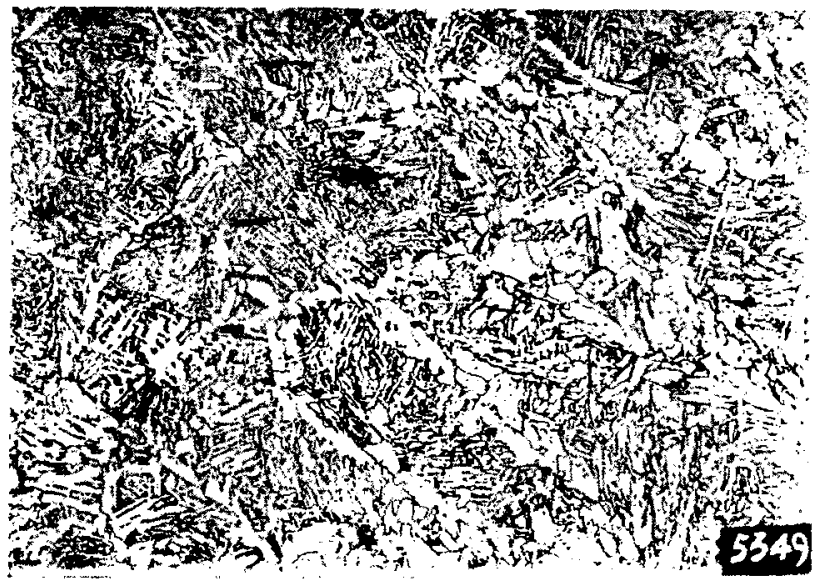

\%ox: me ruasitron

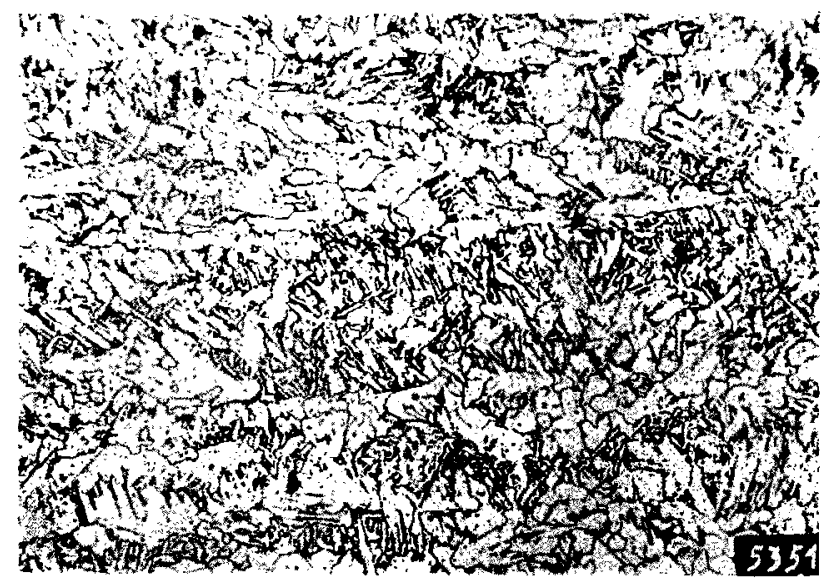

Sinturtur.

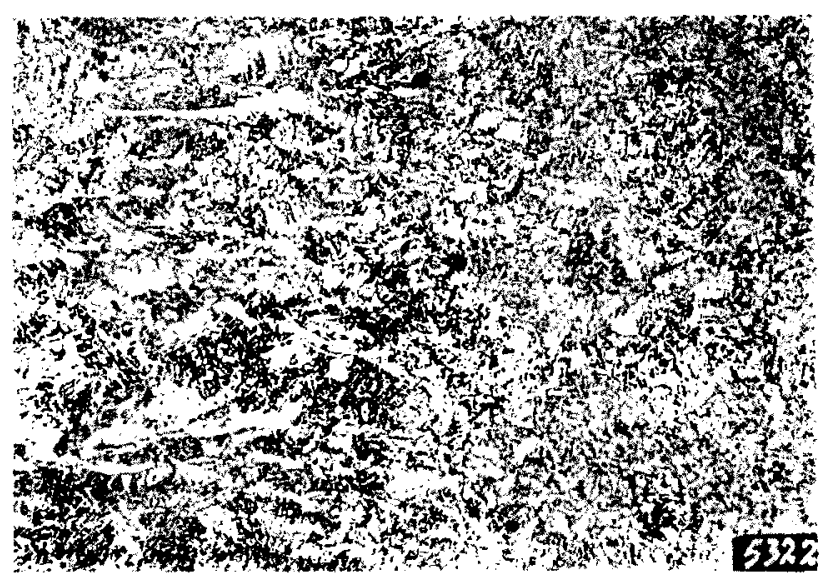

Zoxe bl: rhasstox

EAAMENS MCHOGHAPHIQLES

bomnes pour les besoine d'une construclion normale. D'autre part, lallongement augmente lorsque la tempéature d'épreuve s'abaisse. Quant aux effets de vieillissement, ceux-ci angmentent jusqu'à un éconissage de 3-4\%: ils deviennent ensuite constants et sont ligerement reduits pendant que la température d'essai est abaissée.

Les diagrammes relatifs à la résilience ont des aspects semblables a ceux de l'allongement avec des décroissances plus acentuées en fonction du degre d'ecrouissase of des lemperilures dienoissantes. L'influence du vicillissement jusqu'alux déformations de l'ordre de 3 \% et jusqu'aux températures de lordre de $-10^{\circ} \mathrm{C}$ est tres faible; elle ne devienl apparente quiax fabbles de-

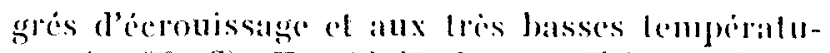
res (-30" C). En depit des conditions d'essiti defarorables, les résiliences considerés restent pleinement salisfaisantes. A de tres basses tompéralures d'épreuve, les résilienees, apres écontissatge de $10 \%$ et vieillissement artificiel, sont riduites de moins de $50 \%$ el cela apporle une conclusion favorable quant at non-vieillissement de l'acier COF. 3. Par ailleurs, on a remarqué un accroissement inallendu de l'indier do qualliti:

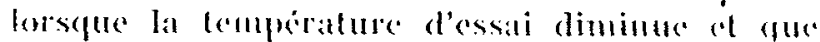
dans les conditions les moins favorables londice de qualite est fres salisfaisant. Les diatgrammes sus-mentionnes somt reprodults ei-eontre and des tableatux numerotio de 1 a $\mathrm{IX}$. 


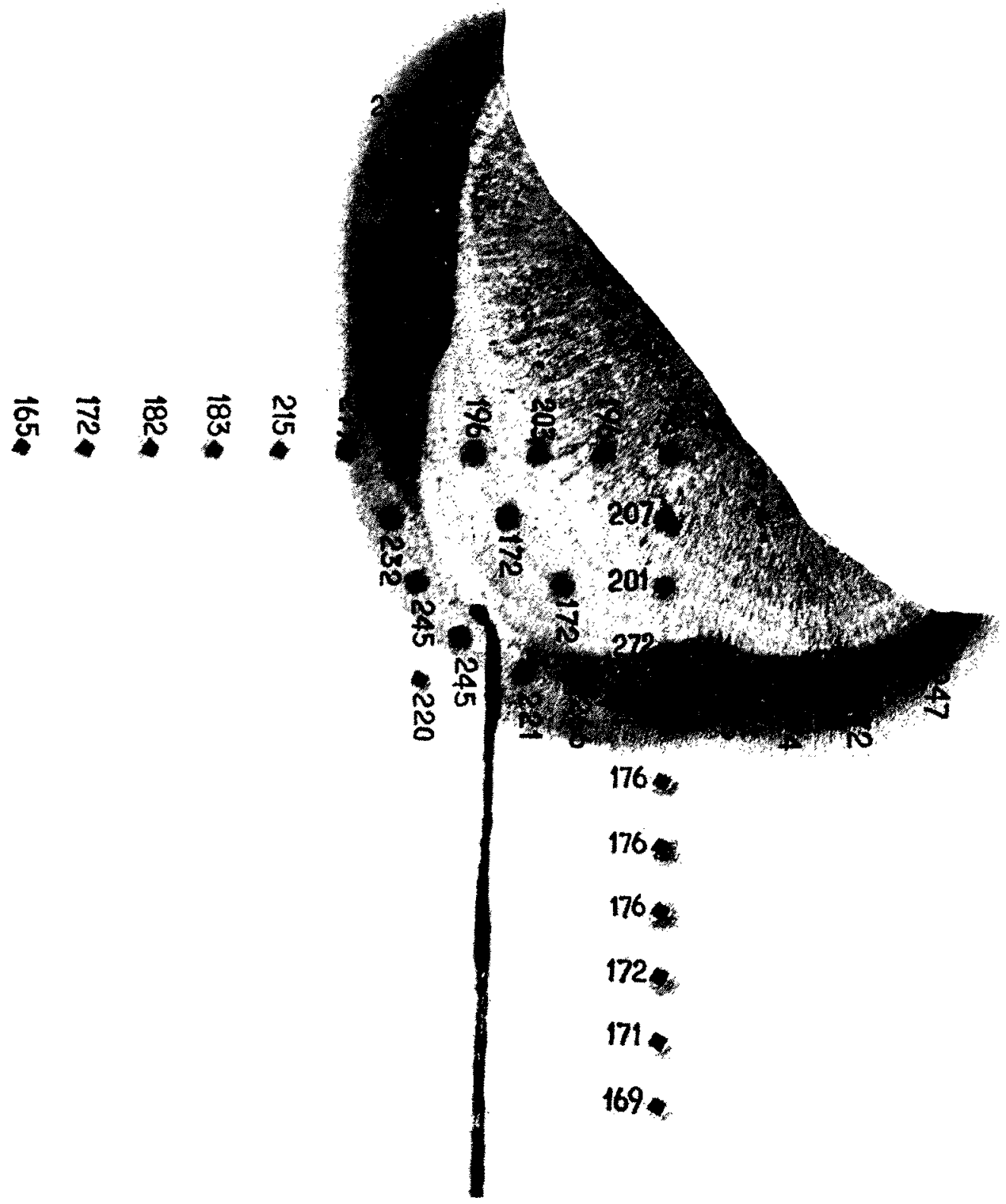

Essai de félure fait sur une tôle COF.3 normalisée. Epaisseur $12 \mathrm{~mm}$. Soudures à la main, 


\section{Influence de la température d'essai et de l'écrouissage sur les caractéristiques de l'acier COF.3.}

liprourelles prises sur libles normalisces de 19 a 20 mm d'eparisseur

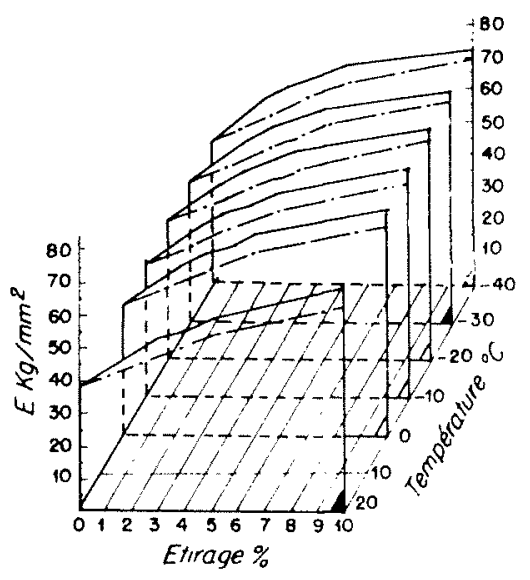

LIMTE B.ASTlote

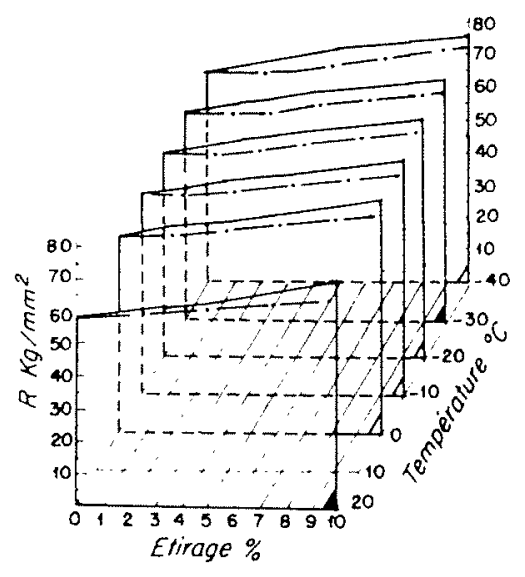

RESTraver a LA RTPTERE

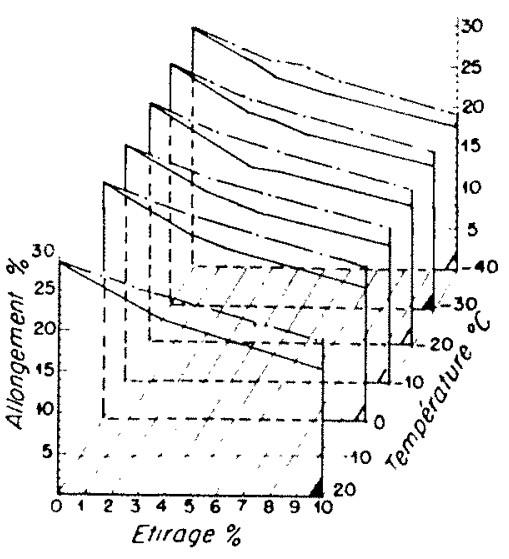

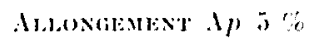

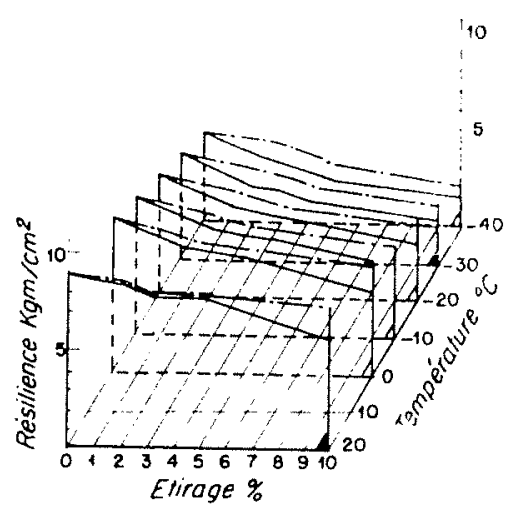

RESALILNCE

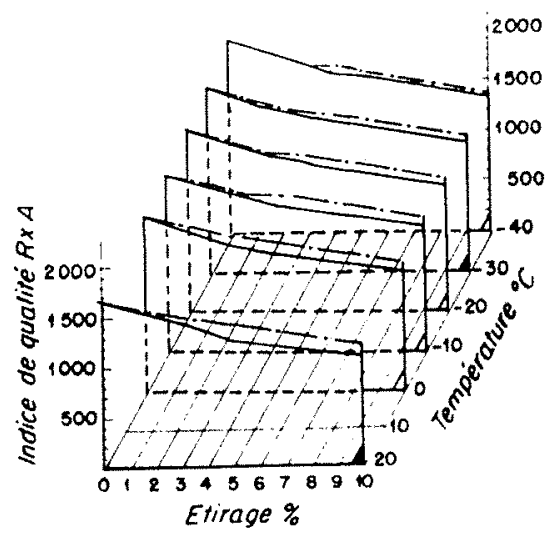

INDICE DE QUALT: $\mathrm{l} \times \mathrm{A}$

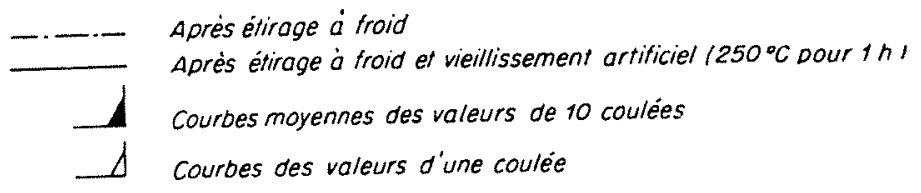


TABLEAU VII

Essais de pliage à froid sur tôles COF.3 de 28 à $30 \mathrm{~mm}$ d'épaisseu: de $150 \times 400$ de tôles de différentes coulées pliées à branches parallèles sur $3 \mathrm{e}$

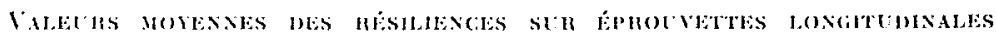

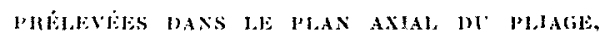

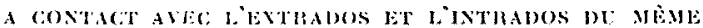

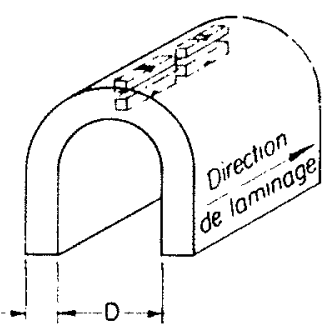

\begin{tabular}{|c|c|c|c|c|c|}
\hline Rief. & Posilion des essuis & Elal all melal & $\begin{array}{l}\text { Valeurs } \\
\text { moyennes } \\
\text { de k } \\
\text { kgmicm: }\end{array}$ & $\begin{array}{l}\text { Dimis } \\
\text { lotules }\end{array}$ & $\begin{array}{l}\text { tions } \because \\
\text { par repport } \\
\text { a l'étut } \\
\text { precédent }\end{array}$ \\
\hline 1 & Avanl ke pliage & Normalise & 9,13 & $\cdots$ & $\cdots$ \\
\hline 2 & Dans lextraclos & Normalise el cliré a froid & 6,33 & $30 \%$ & - \\
\hline 3 & Dans lextrados & Normalise et etire a froid & $\therefore, 50$ & $5 \%$ & $311 \%$ \\
\hline 4 & Dans l'intrados & Normalise et refoule a froid & 8.60 & a $\%$ & $\cdots$ \\
\hline 5 & Mans l'intrados & $\begin{array}{l}\text { Normalise refoule a froid el } \\
\text { vieilli arliticiellement }\end{array}$ & $\therefore, 16$ & $4: 3 \%$ & $111 \because$ \\
\hline
\end{tabular}

TABLEAU VIII

TABLEAU IX

\section{Caractéristiques mécaniques à températures élevées} des tôles d'acier COF.3 normalisées et adoucies.

(Valeurs moyennes sur 10 coulees)

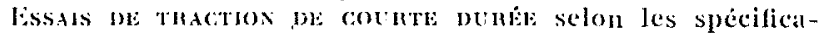
lions D.I.N.1602. Fossais do viscosite selon les spécificalions DIN-A-11i.

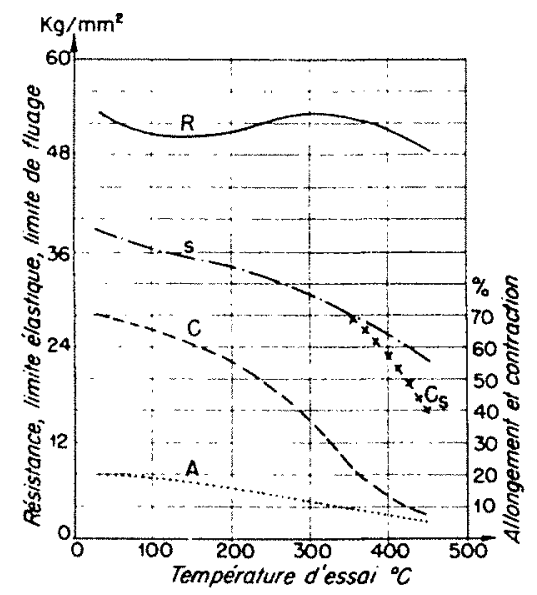

$\begin{array}{ll}\mathrm{R} & \begin{array}{l}\text { Résistance a la rupture } \\ \text { Limile elastique }\end{array} \\ \ldots \ldots+\ldots+\mathrm{C}_{\mathrm{s}} & \text { Limife de fluage } \\ \ldots \ldots \ldots+\mathrm{C} & \text { Contraction } \\ \ldots \ldots \ldots+\mathrm{A} & \text { Allongement }\end{array}$

Valeurs de la limite de sécurité $Y$ de différents aciers

Comme specifie par l'assuciation Nathonale de Conthole: de Conmestion, Rome.

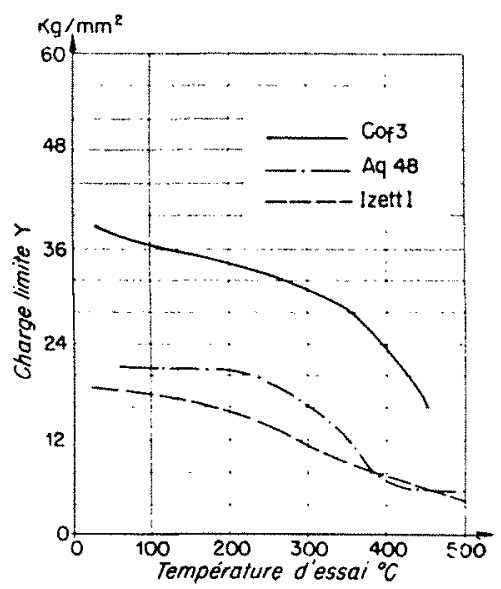




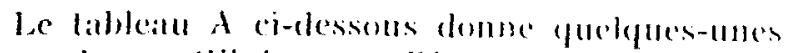
des valeurs ulilisces pour l'établissenunt des diagrammes mentionnés. Cos valeurs représentont

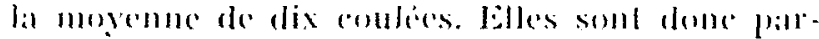
liculierement significatives.

TABLEAU A

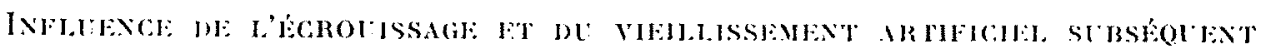

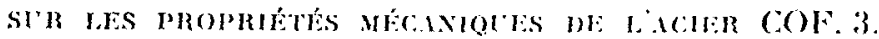

Caractéristiques mécaniques moyennes de triles normalise'es de 9 a $26 \mathrm{~mm}$ d'epaisseur probronal de jo roulées.

Essais à température ambiante

\begin{tabular}{|c|c|c|c|c|}
\hline EYTAT & 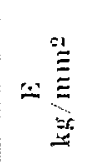 & $\simeq \underset{z}{\stackrel{3}{\Xi}}$ & $7::^{\circ}$ & 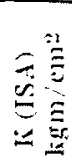 \\
\hline Normalisces $\ldots \ldots \ldots \ldots$ & 37.8 & 38, & 28,4 & 11,4 \\
\hline 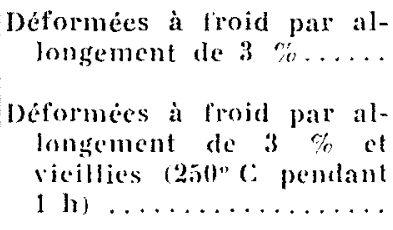 & $\pi 2,7$ & 61,7 & 23 & 9.3 \\
\hline 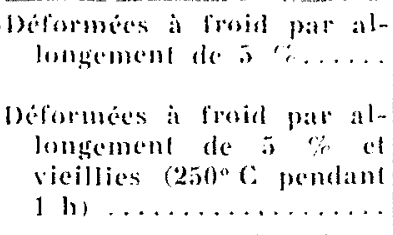 & 38.8 & (ii), & 20.1 & 0.3 \\
\hline 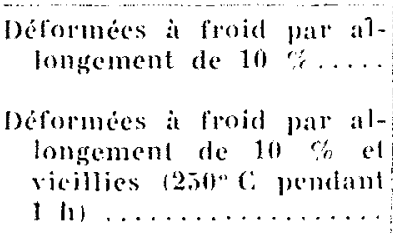 & (i巳,? & 64,7 & 15,8 & 8,9 \\
\hline
\end{tabular}

Dans les constructions somdées, non seulement le métal de hase, mais également les joints soudés peurent être sommis à l'action de l'écrouissage a froid, du finil des deformations a froja qui sont la consequence des procédés de fahrication spéciaux en matière de construction de conduites forcées, des effels de retrait dus a lat soudure et des adaptalions sous charge.

Dans le but de controler cel aspect de la question, des ichantillons furent prépares avee des loles de $26 \mathrm{~mm}$ d'épaisseur appartenant à 5 conlées différentes. Les échantillons furent soudés par rapprochenent a la main en utilisant des dectrodes enrobés basiques. De ces ichantillons des éprouvettes furent prises a travers la sou-
Essais à température de $30^{\circ} \mathrm{C}$ au-dessous de zéro

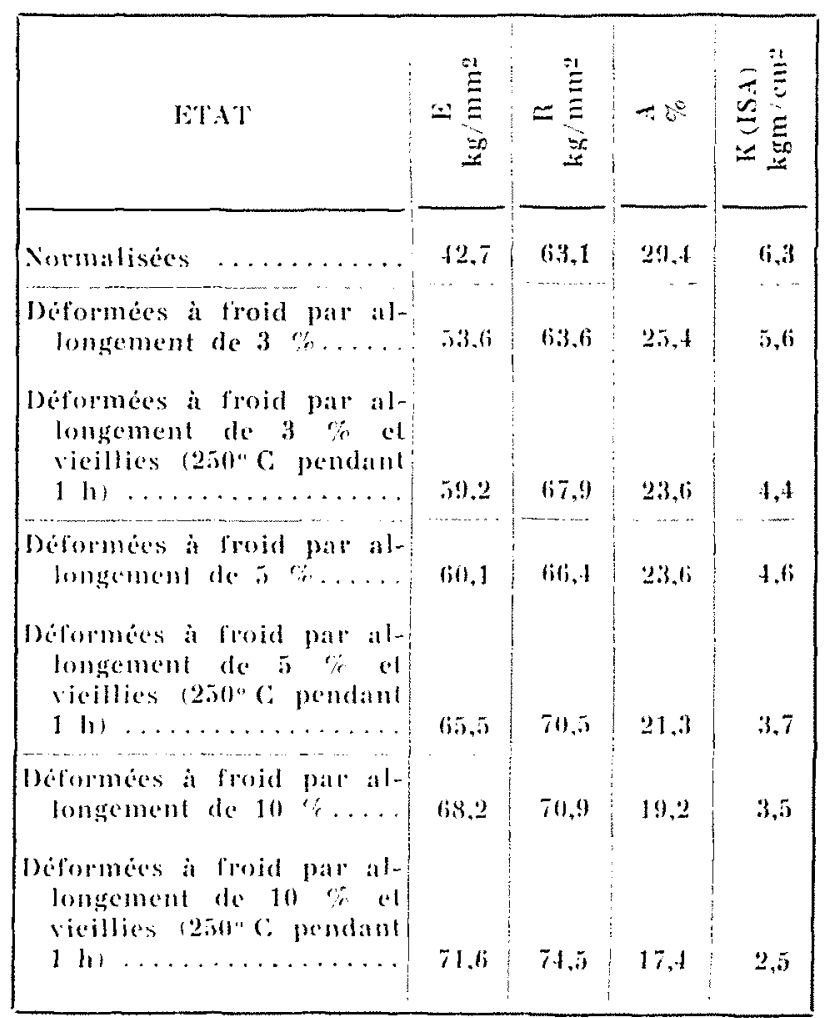

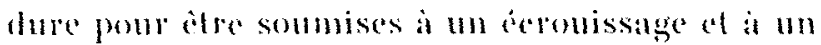
vieillissement arlificiel. Ces eprouvelles furent deformees a froid par allongement jusqu'à 5 g ansi gue celat se probluit dans la fabrication des luyaux suppressés a.. ef utilisces pour la préparalion d'eprouvelles de traction of de choc. bes resultats moyens sous diverses conditions dessai sont indigues dans le tablean b ci-apreses coll comparaison avec les resullats do la mome sorle d'iprenves failes sur le molal de base des lobes des cind mimes coules. Dans ce tableat on jeul noter dans les deux cas une grande simililude de lécounissage a froid el du vicillissoment artificiel sur E et R. Plus frononere est cependant l'influenee des facteuts di-dessus sur 
les illongements des éprouselles somderes. Common pour le choc, les ćprouveltes souties semblent atre moins alleclies que les toles. En tout cas, les proprictis de choc et de traction sont tout a fait sulfisanles pour assurer de larges malges de securite, el le lableau complet apparait satisfaisant quand on l'examine de ec point de vue particulice.

\section{TABLEAL $B$}

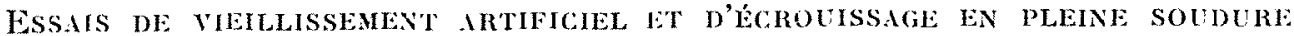

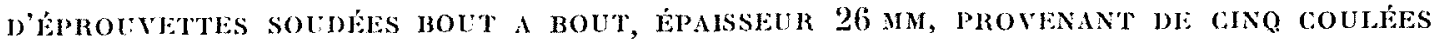
Valeurs moyennes de caractéristiques mécaniques d'éprouvettes recuites it $650^{\circ} \mathrm{C}$.

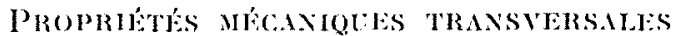 \\ D) QIELOLES TOLES NORMALISËES

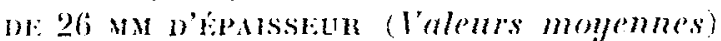

Essais à température ambiante éprouvettes de choc prises dans le milieu de la soudure

\begin{tabular}{|c|c|c|c|c|}
\hline ETAT & 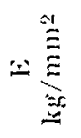 & 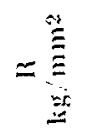 & Tis & 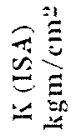 \\
\hline Normalisies $\ldots \ldots \ldots \ldots$ & 39,1 & 56,6 & 21,4 & 15 \\
\hline 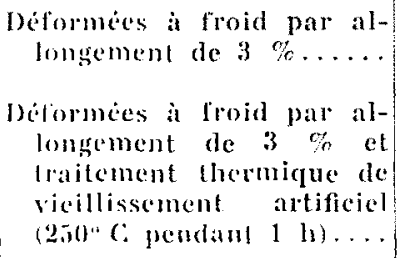 & $49, \bar{i}$ & 5 & 18,9 & 11.4 \\
\hline 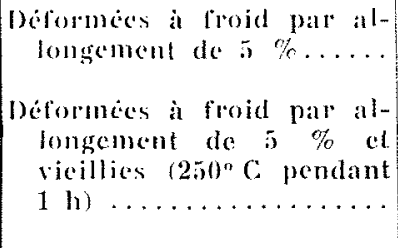 & $5,3,0$ & 61.4 & $1, \pi, 3$ & 12,7 \\
\hline
\end{tabular}

Les resultats obtenus fors de l'ulilisalion de lacice COF. 3 ont conduit leurs auteurs à ('n anvisiger l'usage a d'autres emplois.

Il leur paraissait intéressant pour cela de vérilier l'aptitude de cet acier aux constructions d'appareils thermiques el chimigues en vue de profiter de ses hautes qualités de traction associées ì celles de leur non-vieillissement.

On a done fait des essais pour determiner les proprietés mécaniques de eet acier à des tempiratures devées sous traction de courte duree et des essais de viscosile. (es essais, trop peu nombreux pour conduire a des conclusions statistiques définilives, sont eqpendant suffisants dans un cadre dorientation el de comparaison appoximalive aree dautres aciers habiluellement utilisés pour cet objel.

Le's risultats de lels essais sont rapportés en des tableaux oi l'on examine les valleurs de li-
Essais à température ambiante

\begin{tabular}{|c|c|c|c|c|}
\hline E1.1T & $\leftarrow \stackrel{\substack{\Xi \\
\Xi}}{\Xi}$ & 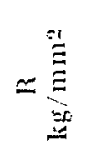 & +0 & 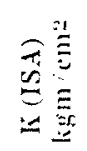 \\
\hline Normalisces ... & $3 \overline{7}, \bar{i}$ & 55,5 & 29,6 & 11,6 \\
\hline $\begin{array}{l}\text { Deformces à froid par al- } \\
\text { longement de } 3 \text {. } \% \text {. }\end{array}$ & $44, \pi$ & $5 \pi, 1$ & 26,3 & 10,1 \\
\hline 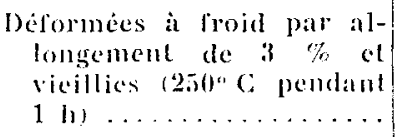 & (201) 3 & 59. & 23.7 & 9.7 \\
\hline 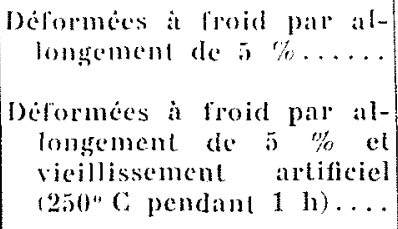 & $51, \overline{5}$ & 61,3 & 20,9 & 10,1 \\
\hline
\end{tabular}

mite de sécurite spécifiées par l'A.N.C.C. Celles-ei sont comparées avec celles spécifièes par la mème organisation pour un acier de chaudiere du type Aq 48 UN 815 avec $R 48$ a $55 \mathrm{~kg} / \mathrm{mmm}^{\prime \prime}$ an qu'avec l'acier IzeTr I sans vieillissement de KRUpp. Ces comparaisons mettent en ividence les excellentes qualités de l'acier COF. 3.

Nous avons nous-mêmes complété les essais de M. Fornacr par des essais effectués d'apres les principes de M. Schnadt sur lacier type « ConlOOR E $\triangleq$ que nous utilisons habituellement en France sur les conduites forces, notamment pour lat fabrication des tuyaux surpresses.

Une lole d'acier nuance « CoNron $\mathrm{E} »$, a chrome-cuive manganese, de $12 \mathrm{~mm}$ d'epaisseur, élaborée suivant les spécifications techniques du Marché et Cahier des Charges de la Sociele Hydrotechnique de France pour la Fourniture et lo 
Montage des conduites forcées en miall el de leurs accessoires, a ditecuite a 850 " C puis découpée a froid en :

8 iprouveltes de 1 raction de section $50 \times 12 \mathrm{~mm}$, repérées $A 1, A 2, \cdots-B 1, B 2,-C A, C 2$, D1, D2, et :

5 plaques de $200 \times 160$ repérées deux fois : E1. E2, E3, Et et une fois (A1, G2 voir tig. 1 cirdessous).

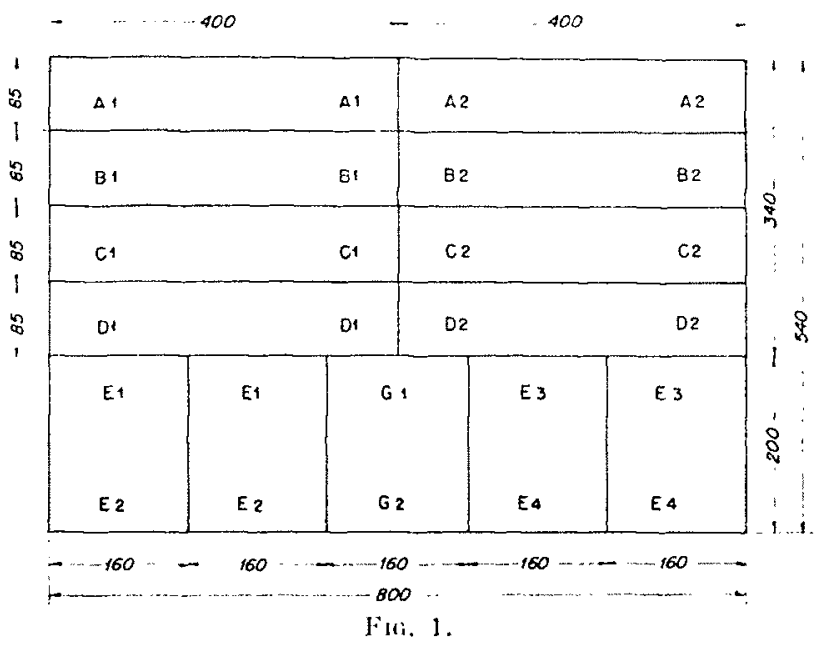

Eehantillon tible Confon le, epaisseur $12 \mathrm{~mm}$. Selis du laminage $\rightarrow$

Les éprouvettes de tratction onl subi les allongements permanents suivants, mesurés sur une base de 100 mum (voir fig. 2 ci-dessous) :
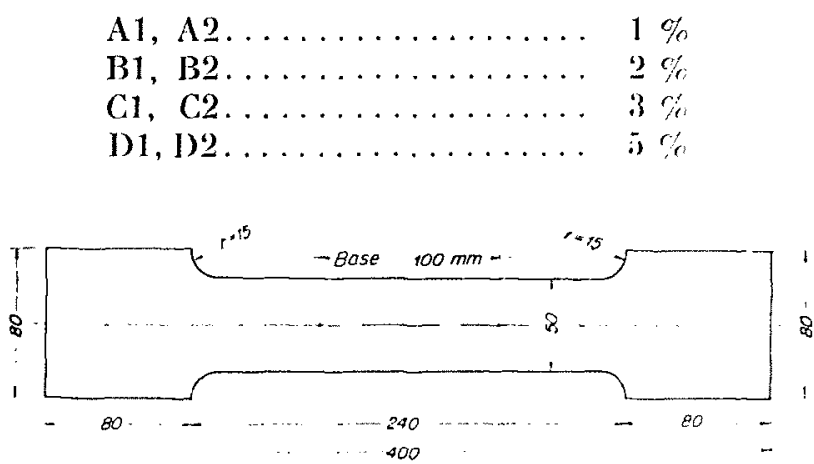

Fili, 2.

Eppouvetles de traction $A, B, C, D$.

Les plaques de $200 \times 160$ ont été soudées deux par deux :

E1, E2 : soudure main, ilectrode Marine 55.

E3, E4 : soudure main, électrode Marine 45.

Ces soudures nont subi aucun traitement thermique après leur exécution. La plaque G1, G2 reste représentative de lacier normalisé.

'Trois éprouveltes dénommées, par M. Scinsot,

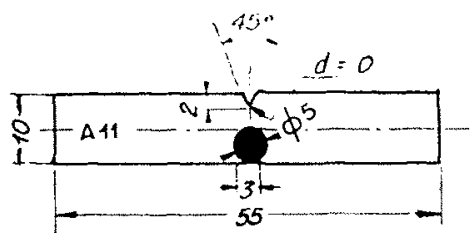

liprouvelle cohéracic

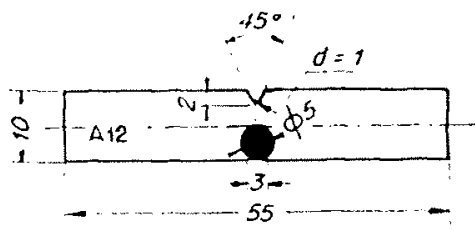

Liprouvette resilacio

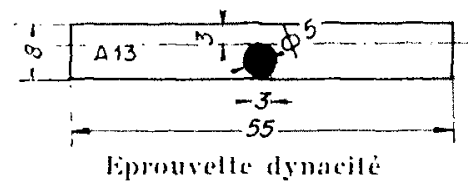

Fiti. 3

ErRovvettes Somnate

de cohéracie (Ko), résilacie (K1), dynacité (Ki), (voir fig. 3 ci-dessus) ont éte pritevées dans chacun des échantillons ci-clessus définis (voir fig. $f$, 5, 6 ci-apriss et ont permis de tracer les diaarammes de choc de l'acier "Coxvor $\mathrm{E}$ » (roir (ig. 7).

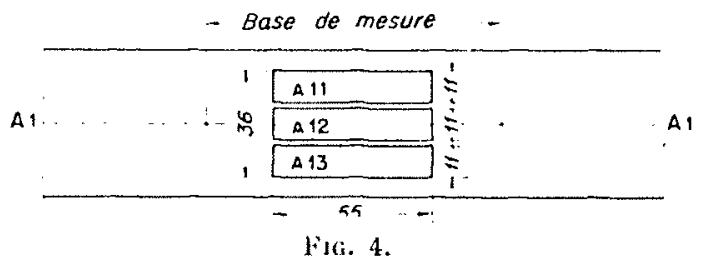

Position des éprouretles de coheracie, resilacie, dynacili ditns les iprouvettes de traction A, B, C, D).
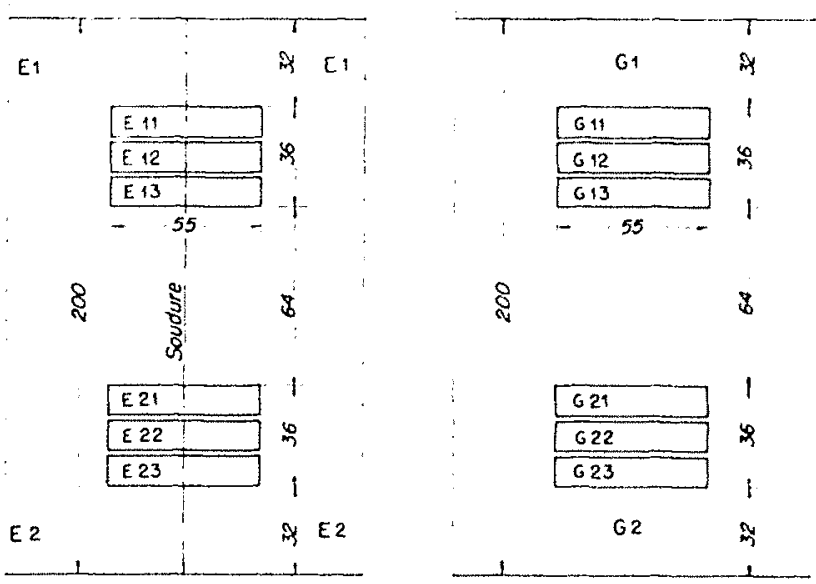

Position des eprourelles de colderacie, resilated dynacite, dans:

Fin. 5.

Fia. 6.

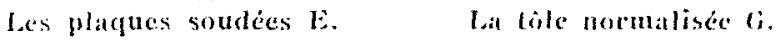




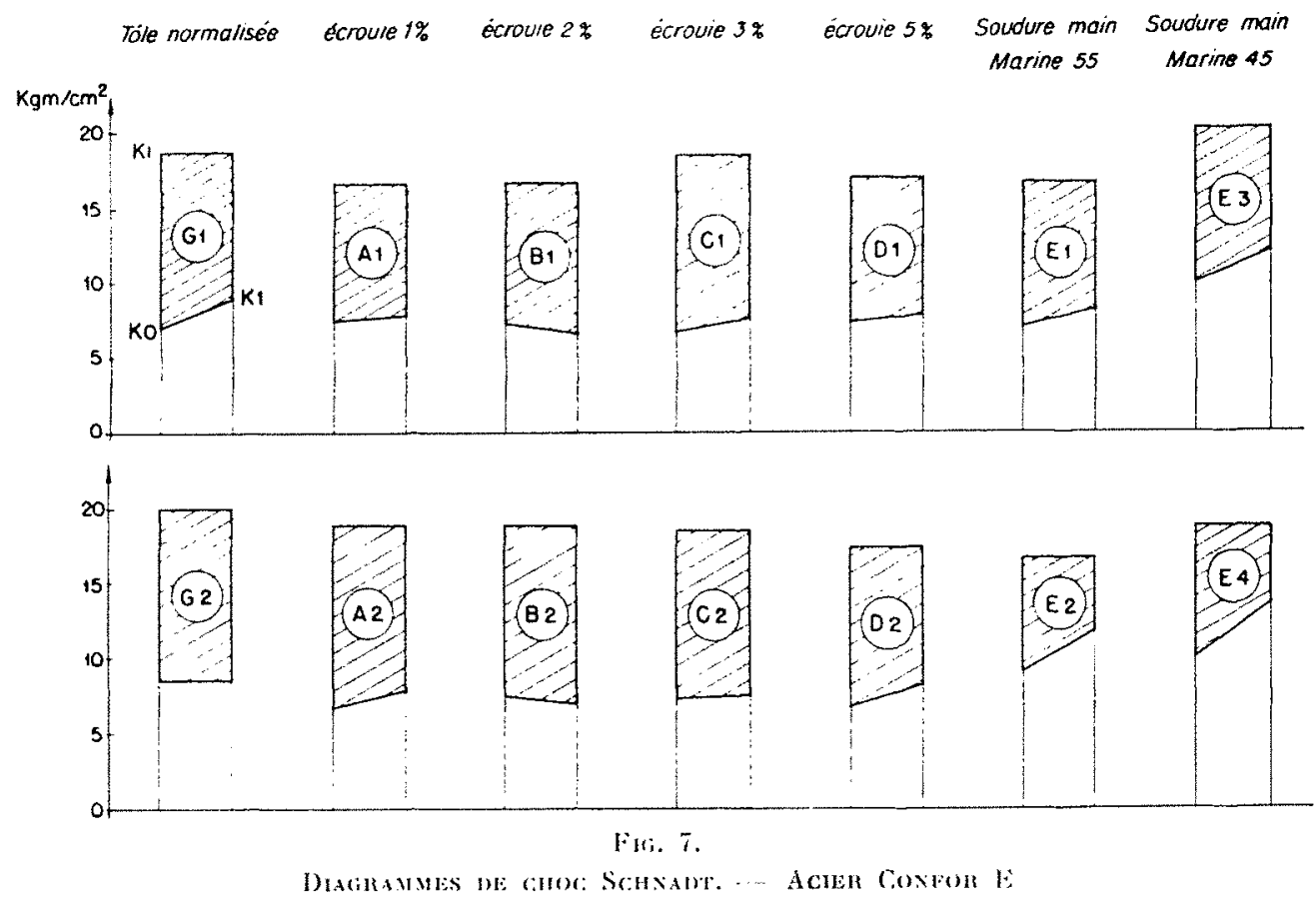

L'examen de ces diagrammes montre :

Qu'un écrouissage de l'acier « Coxpon $\mathrm{E}$ » de $1,2,3 \%$ n'entraine pas d'augmentalion de la fragilite de l'acier;

Que laugmentation de fragiliti apportie par un ecrouissage de 5\% est tres faible;

Que la soudure exécutéc avec lélectrode Malrine 45 est plus résiliente que celle faile avee l'électrode Marine $5 \overline{5}$, cependant plus résistante.

Il n'est pas donteux que de tels aciers utilisess a la fabrication des luyaux equipant les plus importantes conduites forcés modernes alimentant sous hautes chutes les plus puissantes usines hydro-électriques d'Italic et de France sont tout à fait remarquables. Les operations d'auto-frettage et de surpressage ne les rendent pas fragiles et ils donnent une sécurité exeeptionnelle aux ouvages soudés sur lesquels ils sont utilisés.

Mais ee qui compte avant tout of plus que tout, ee sont les essatis systematiques sous pression hydraulique auxquejs sont soumis les fuyaux surpressés au dernier stade de leur fabrication.

C'est pourquoi nous prions nos lecteurs de bien rouloir se reporter à ee que nous arons écril i colle occasion dans un arlicle intilule : $* A$ propos d'un accident survenu à une conduite apress quarante ans d'exploitation $\gg\left({ }^{*}\right)$.

Et pour terminer nous n'hésitons pass à repro-

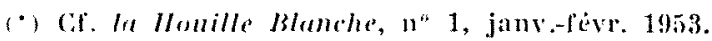

duire in extenso certaines des importantes conclusions que nous avions tirées :

"On peut tout d'abord affirmer que lemploi des tuyaux surpressés et des tuyaux auto-frelles surporessés dome à l'exploitant la sécurite la phus complete qu'il soit possible d'oblenir en matiere de conduites forcées. Le coefficient de sécuriti est vérifié expérimentalement par rapport à î limite éastique rélle du métal utilisé, qui, on te sait, est toujours supérieure au minimum théorique, e'est-it-dire que le coefficient de sceurili récl est loujours suprericur au coefficient de securité théorique.

"On peut également affirmer que pour les tuyaux surpressés et les luyaux auto-frettes surpressés, il ny a aucun inconvénient à réduire le coefficient de sécurite théorique admis jusqu’a maintenant jusqu'au minimum compatible avec l'épreuve au double, c'est-à-dire : 2 .

"Pour les tuyaux simplement soudés, une sécurite du même genre ne peut ètre obtenue qu'aulant que l'éprouve hydraulique est poussée jusqu'à alteindre une déformation plastique de 0,5 i 1 \% en construisant, ainsi que nous avons déjà 'u l'oceasion de le faire pour une importante conduite forcés en Italie, des tuyaux à « limite élastique el coefficient de sécurité contrôlés».

"Cela permet d'envisager pour les tuyaux soudés la réduction jusqu'à 2 du coefficient de sécurité, comme pour les tuyaux précédents, et de réaliser ainsi de nouvelles et importantes économies de métal. » 